\title{
Caracterização do solo de cobertura de aterros encerrados com ferramentas (geo)estatísticas
}

\section{Characterization of soil covers in closed landfill sites with (geo)statistical tools}

\author{
Alessandro Samuel-Rosa \\ Engenheiro Agrônomo. Aluno do curso de Mestrado do Programa de Pós-Graduação em Ciência do Solo da Universidade Federal de Santa Maria (UFSM). \\ Bolsista do Conselho Nacional de Desenvolvimento Científico e Tecnológico (CNPq).
}

Ricardo Simão Diniz Dalmolin

Engenheiro Agrônomo. Professor Doutor no Departamento de Solos do Centro de Ciências Rurais (CCR) da UFSM. Pesquisador do CNPq.

\section{Fabrício de Araújo Pedron}

Engenheiro Agrônomo. Professor Doutor no Departamento de Solos do CCR da UFSM.

\section{Resumo}

Inúmeros trabalhos abordam a elaboração de estratégias amostrais e a aplicação de ferramentas (geo)estatísticas no estudo de atributos do solo. Entretanto, são escassos os trabalhos envolvendo a aplicação desta abordagem no monitoramento de solos construídos sobre aterros encerrados de resíduos sólidos urbanos. Este estudo mostra que a densidade amostral necessária para tornar possível o uso da geoestatística em tais casos, elevaria os custos operacionais. A melhor alternativa é a utilização dos métodos de estatística multivariada (análise de componentes principais e de agrupamento) para definição de zonas homogêneas de manejo. Os atributos que melhor explicam a estrutura da variabilidade do solo construído são o teor de areia (ou argila), a saturação por bases e o pH, todos relacionados com a contaminação do solo com chorume e o adequado desenvolvimento da vegetação.

Palavras-chave: resíduos sólidos urbanos; solos construídos; malha amostral; zonas de manejo.

\section{Abstract}

Several studies address the development of sampling strategies and implementation of (geo)statistical tools in the study of soil properties. However, there is a lack of studies in the application of such approach to monitor soil covers in closed landfill sites of urban solid waste. This study shows that the sampling density needed to make possible the use of geostatistics in such cases would raise operational costs. The best alternative is the use of multivariate statistics methods (principal components and cluster analysis) to define homogeneous management zones. The soil attributes that best explain the structure of soil variability are sand (or clay) content, base saturation and pH, all related with soil contamination by leachate and with the proper development of vegetation.

Keywords: urban solid waste; constructed soils; sampling grid; management zones. 


\section{Introdução}

Quando a capacidade de um aterro receber novas quantidades de resíduos sólidos urbanos (RSU) é esgotada, procede-se ao seu encerramento. Nesta fase, os RSU devem receber uma cobertura de solo que os isole efetivamente, reduzindo os fenômenos de erosão e infiltração, e devem proporcionar o desenvolvimento da biota (DWYER, 2000). O processo de constituição de um ambiente pedológico, formado por materiais e procedimentos antrópicos, origina o que se chama de solo construído, o qual passa a ter uma evolução genética e a atuar no ecossistema de maneira diferente daquela de ambientes inalterados (KÄMPF; SCHNEIDER; GIASSON, 1997). O principal responsável por esse comportamento é o novo material de origem formado (DUDAL; NACHTERGAELE; PURNELL, 2002), no qual a decomposição microbiológica é o processo dominante, resultando na formação de gases e chorume (FRANTZIS, 1991). A estabilização do solo construído como componente ambiental pode levar décadas, pois a decomposição dos RSU continua por vários anos após o encerramento.

A adoção de práticas de reabilitação nos aterros encerrados é a forma mais eficiente de auxílio à estabilização do solo construído e sua introdução à dinâmica do ecossistema. Dentre as práticas mais recomendadas, destaca-se a caracterização espacial dos atributos do solo construído. Para isso, é fundamental elaborar uma estratégia amostral capaz de caracterizar, efetivamente, a variabilidade contida na área, a partir de um número restrito de observações (MCBRATNEY et al., 1999; STEIN; ETTEMA, 2003). Inúmeros trabalhos têm abordado a elaboração de estratégias amostrais apropriadas e a aplicação de ferramentas geoestatísticas no estudo de atributos do solo (WEBSTER; OLIVER, 1992; KERRY; OLIVER, 2004). Entretanto, são escassos os trabalhos envolvendo a aplicação desta abordagem no monitoramento de solos construídos (CRITTO; CARLON; MARCOMINI, 2003; GUILLEN; HERTZOG, 2004), o que dificulta a elaboração da estratégia amostral, haja vista a sua heterogeneidade. Na maioria dos casos, esses solos são caracterizados por meio de valores médios e nãoespacializados, o que dificulta a elaboração de um projeto adequado para a reabilitação da área.

O objetivo deste trabalho foi avaliar a qualidade do solo construído em um aterro encerrado de RSU, caracterizando o comportamento espacial de seus atributos. Considerando o potencial de uso das ferramentas (geo)estatísticas no monitoramento geotécnico e ambiental de solos construídos, são definidos parâmetros para subsidiar a amostragem de ambientes pedológicos similares com máxima eficiência e custo mínimo.

\section{Metodologia}

O estudo foi desenvolvido na antiga área de descarte de RSU de Santa Maria, RS (29³9'43"S; 5352’30”W). O clima regional é do tipo Cfa II, com temperatura e precipitação pluviométrica média anuais de $19,2^{\circ} \mathrm{C}$ e $1.708 \mathrm{~mm}$, respectivamente (MORENO, 1961). No interior da área, são encontrados três aterros encerrados, sendo avaliado aqui o mais antigo, que foi operado entre 1982 e meados da década de 1990. Durante este período, os RSU gerados no município eram coletados pela Prefeitura Municipal e depositados sobre o solo em condições naturais. A partir da segunda metade dos anos 1990, o serviço de recolhimento de RSU foi terceirizado, quando os RSU do antigo aterro receberam uma cobertura com solo da área de empréstimo, que foi obtido pela mistura do material dos horizontes A, B e C de Argissolo Vermelho, seguido da sua compactação. Atualmente, com superfície total de $31.813 \mathrm{~m}^{2}$ e espessura máxima da massa de RSU de aproximadamente $2,0 \mathrm{~m}, 82 \%$ da área permanece coberta, tendo o restante sido revolvido por catadores para coleta de materiais recicláveis. $\mathrm{O}$ solo construído possui espessura de 0,2 a 1,0m, e após 15 anos do seu encerramento nenhuma intervenção para o estabelecimento de vegetação foi realizada (SAMUEL-ROSA; DALMOLIN, 2009).

A caracterização do solo construído foi realizada pela coleta de 48 amostras na profundidade de 0 a $0,2 \mathrm{~m}$, utilizando grade regular com espaçamento de 22,4m. O solo da área de empréstimo foi caracterizado para fins de comparação. As amostras foram analisadas em triplicata, seguindo metodologia descrita pela Embrapa (1997) Foram determinados: a composição granulométrica, o pH, a acidez potencial $\left(\mathrm{H}^{+}+\mathrm{Al}^{3+}\right)$, os teores de bases trocáveis $\left(\mathrm{Ca}^{2+}, \mathrm{Mg}^{2+}, \mathrm{K}^{+}, \mathrm{Na}^{+}\right)$ e $\mathrm{Al}^{3+}$, fósforo (P) e matéria orgânica (MO). A partir dos resultados, calculou-se a capacidade de troca de cátions (CTC), a saturação por bases (V\%) e por alumínio (Al\%). Os resultados foram analisados via estatística descritiva, e a comparação entre o solo construído e a área de empréstimo foi realizada em termos absolutos, usando a média aritmética e os valores mínimo e máximo. A análise espacial dos atributos do solo construído foi realizada por meio da análise variográfica (ISAAKS; SRIVASTAVA, 1989). O índice de dependência espacial (IDE) foi utilizado para verificar a dependência espacial. A partir destas informações, foram elaborados os mapas de distribuição espacial dos atributos do solo. A análise de componentes principais (ACP) foi usada para escolha das variáveis com maior poder explicativo da variabilidade do solo. Essas variáveis foram utilizadas na análise de agrupamento. O número final de agrupamentos foi determinado pela ocorrência de soluções similares entre algoritmos, além da necessidade de se obter um resultado com significado prático. Cada agrupamento foi analisado por meio de estatísticas descritivas.

\section{Resultados}

O solo construído apresenta maiores teores de areia, bases trocáveis e $\mathrm{MO}$ e menores teores de argila, $\mathrm{Al}^{3+}$ e $\mathrm{H}^{+}+\mathrm{Al}^{3+}$ quando comparado ao solo da área de empréstimo (Tabela 1). Atributos como $\mathrm{pH}, \mathrm{CTC}, \mathrm{V} \%$ e P continuaram semelhantes, apresentando alguns aumentos localizados. No caso das bases trocáveis, destaca-se o teor médio de $\mathrm{Ca}^{2+}$, duas vezes superior, e o teor máximo, quatro vezes 
superior. Para $\mathrm{K}^{+}$e $\mathrm{Na}^{+}$, os teores médios são, respectivamente, duas e sete vezes maiores e os teores máximos, 10 e 35 vezes superiores.

Os atributos do solo construído apresentaram alta variabilidade (exceto areia e argila) (Tabela 2). Fatores como alto CV\% e IDE, distribuição não-normal, baixos coeficientes de determinação $\left(R^{2}\right)$ no ajuste dos semivariogramas, pequeno alcance e ocorrência de efeito proporcional prejudicaram a detecção da estrutura da variabilidade espacial da maior parte dos atributos. Destaque é dado ao alcance médio observado de $50,2 \mathrm{~m}$, variando entre 32,2 e 133,2m, respectivamente, para V\% e argila. A Figura la e b mostra o mapa de distribuição espacial dos teores de areia e argila pelo método do inverso do quadrado da distância (IQD).

Os atributos do solo teor de areia, $\mathrm{V} \%$ e $\mathrm{pH}$ foram aqueles que apresentaram pesos mais significativos nas três primeiras componentes principais (CP) extraídas. Estas CP concentram 95,13\% da variância total explicada. A análise de agrupamento utilizando estes atributos resultou na formação de dois agrupamentos $\left(G_{1}\right.$ e $\left.G_{2}\right)$ (Figura 1c). $G_{1}$ possui teores mais elevados de $\mathrm{Al}^{3+}, \mathrm{H}^{+}+\mathrm{Al}^{3+}$ e argila, enquanto $\mathrm{G}_{2}$ possui teores mais elevados de $\mathrm{K}^{+}, \mathrm{Na}^{+}$, $\mathrm{P}$ e areia, além de maior $\mathrm{pH}$. Em ambos, os teores de $\mathrm{Ca}^{2+}, \mathrm{Mg}^{2+}$ e MO são similares (Tabela 3).

\section{Discussão}

O menor conteúdo de argila no solo construído indica a ocorrência de processo de enriquecimento com areia. A mistura do material dos horizontes do solo utilizado na cobertura dos RSU e a erosão devem ser os principais responsáveis.
Os valores mais elevados de pH no solo construído são resultado do efeito alcalino do chorume gerado durante a decomposição dos RSU, que possui pH entre 7,8 e 8,2 (LAUERMANN, 2008). Essa interação é possível devido à saturação do solo com chorume em alguns pontos da área, especialmente onde a espessura é menor. Todavia, é desejável a ocorrência de pH entre 4,5 e 5,5, faixa considerada ótima para o desenvolvimento das bactérias metanotróficas (DEDYSH; PANIKOV; TIEDJE, 1998). Essas bactérias consomem o metano gerado durante a decomposição dos RSU, convertendo-o em água, dióxido de carbono e biomassa celular, o que reduz a contribuição do aterro para o efeito estufa aumentado (TEIXEIRA et al., 2009).

No caso das bases trocáveis $\left(\mathrm{Ca}^{2+}, \mathrm{K}^{+}, \mathrm{Na}^{+}\right.$e $\left.\mathrm{Mg}^{2+}\right)$, o efeito do chorume é mais efetivo do que em relação ao $\mathrm{pH}$, haja vista os maiores teores encontrados no solo construído (exceto $\mathrm{Mg}^{2+}$ ). Contudo, ao contrário do $\mathrm{Ca}^{2+}$ e $\mathrm{K}^{+}$, o aumento do teor de $\mathrm{Na}^{+}$apresenta efeitos negativos, especialmente pela toxidade às plantas e redução da mineralização da $\mathrm{MO}$, levando à diminuição da disponibilidade de nutrientes, além da dispersão da fração argila (SANTOS; MURAOKA, 1997; CHAVES et al., 2005). Isso contribui para a perda seletiva de argila nos pontos em que o solo permanece saturado com chorume.

A dificuldade de detecção da estrutura da variabilidade espacial dos atributos do solo indica uma distribuição muito heterogênea. Isso é reflexo da forma de deposição do material de cobertura dos RSU, o que compromete a utilização da geoestatística. $\mathrm{O}$ alcance superior a duas vezes o intervalo amostral obtido para alguns atributos do solo indica adequabilidade da estratégia utilizada (KERRY; OLIVER, 2004). Era

Tabela 1 - Atributos do solo da área de empréstimo (horizontes A, B e C) e do solo construído

\begin{tabular}{|c|c|c|c|c|c|c|c|c|}
\hline \multirow{2}{*}{ Local } & Areia & Silte & Argila & \multirow{2}{*}{$\mathrm{pH}$} & MO & V\% & $\mathrm{Al} \%$ & $P$ \\
\hline & & $\mathrm{g} \mathrm{kg}^{-1}$ & & & \multicolumn{3}{|c|}{$\%$} & $\mathrm{mg} \mathrm{kg}^{-1}$ \\
\hline \multicolumn{9}{|c|}{ Área de empréstimo } \\
\hline$A$ & 641 & 168 & 191 & 4,8 & 1,1 & 26 & 54 & 1,0 \\
\hline$B$ & 419 & 140 & 441 & 4,8 & 0,7 & 41 & 47 & 0,3 \\
\hline C & 692 & 187 & 121 & 5,1 & 0,1 & 41 & 53 & 0,2 \\
\hline Média & 584 & 165 & 251 & 4,9 & 0,6 & 36 & 52 & 0,5 \\
\hline \multicolumn{9}{|l|}{ Solo construído } \\
\hline Média & 599 & 162 & 239 & 4,9 & 1,0 & 42 & 39 & 2,1 \\
\hline Mínimo & 471 & 125 & 123 & 4,2 & 0,5 & 25 & 2 & 0,4 \\
\hline Máximo & 751 & 205 & 378 & 7,3 & 1,5 & 94 & 60 & 11,4 \\
\hline Desvio padrão & 70 & 17 & 69 & 0,4 & 0,2 & 12 & 14 & 2,3 \\
\hline \multirow{2}{*}{ Local } & $\mathrm{Ca}^{2+}$ & $\mathrm{Mg}^{2+}$ & $\mathrm{K}^{+}$ & $\mathrm{Na}^{+}$ & $\mathrm{Al}^{3+}$ & $\mathrm{H}^{+}+\mathrm{Al}^{3+}$ & CTC $_{\text {efetiva }}$ & $\mathrm{CTC}_{\text {potencial }}$ \\
\hline & \multicolumn{8}{|c|}{$\mathrm{cmol}_{\mathrm{c}} \mathrm{kg}^{-1}$} \\
\hline \multicolumn{9}{|c|}{ Área de empréstimo } \\
\hline A & 0,5 & 0,8 & 0,08 & 0,01 & 1,6 & 3,9 & 3,0 & 5,3 \\
\hline B & 0,9 & 2,3 & 0,05 & 0,02 & 3,0 & 4,8 & 6,3 & 8,1 \\
\hline C & 0,3 & 1,4 & 0,04 & 0,01 & 1,9 & 2,4 & 3,7 & 4,2 \\
\hline Média & 0,6 & 1,5 & 0,06 & 0,01 & 2,2 & 3,7 & 4,3 & 5,9 \\
\hline \multicolumn{9}{|l|}{ Solo construído } \\
\hline Média & 1,0 & 1,4 & 0,14 & 0,11 & 1,7 & 3,6 & 4,3 & 6,3 \\
\hline Mínimo & 0,5 & 0,5 & 0,03 & 0,01 & 0,1 & 0,4 & 2,4 & 4,0 \\
\hline Máximo & 3,6 & 3,2 & 0,80 & 0,73 & 3,0 & 4,9 & 7,6 & 9,7 \\
\hline Desvio padrão & 0,5 & 0,6 & 0,13 & 0,15 & 0,7 & 0,9 & 1,2 & 1,3 \\
\hline
\end{tabular}


esperado, portanto, que a detecção da estrutura da variabilidade desses atributos fosse favorecida, o que não ocorreu. Em trabalho similar, Campos, Almeida e Souza (2003) utilizaram malha amostral regular (intervalo de 20m) em solo construído sobre mina de carvão a céu aberto, conseguindo detectar apenas a estrutura da variabilidade espacial dos atributos teor de $\mathrm{Al}^{3+}, \mathrm{H}^{+}+\mathrm{Al}^{3+}$ e argila. Os atributos desses solos parecem apresentar variabilidade estruturada em escala menor do que em aterros de RSU, especialmente pela ação acidificante dos resíduos piritosos (SCHAFER; NIELSEN; NETTLETON, 1980; MAÇANEIRO, 2001). Assim, para solos construídos sobre aterros encerrados de RSU, recomenda-se a utilização de pequenos intervalos amostrais. Neste estudo, V\% é o atributo mais restritivo, exigindo distância inferior a 16,1m.

A retenção das frações granulométricas na primeira $\mathrm{CP}$ reflete a maior homogeneidade de sua distribuição espacial, evidenciada na Figura 1. Além disso, esses atributos sofrem pequena alteração ao longo do tempo com o manejo dado ao solo. As demais componentes refletem as condições de fertilidade do solo e o efeito do chorume, ou seja, atributos de caráter dinâmico.

Apesar de possível, a subdivisão da área em mais de duas zonas de manejo foi evitada devido a sua reduzida superfície, o que dificultaria a adoção das ações de intervenção. A solução com duas zonas pareceu a mais adequada. Sua análise mostra que $G_{1}$ englobou as observações com maior teor de argila e menor fertilidade. Em contrapartida, as observações com maior teor de areia e maior fertilidade, nas quais o chorume gerado durante a decomposição dos RSU teve maior influência, foram agrupadas em $G_{2}$. Assim, a melhoria da fertilidade em $G_{2}$ com vistas ao desenvolvimento da vegetação pode ser mais facilitada do que em $G_{1}$. Entretanto, a granulometria do solo em $G_{2}$ representa menor potencial de retenção de água e nutrientes, bem como maior suscetibilidade aos processos erosivos. Quanto aos teores de MO, há necessidade de sua elevação em ambas as zonas de manejo. Essa elevação é fundamental, dadas às funções da MO como promotora da estruturação do solo, retenção de água, capacidade de troca de íons, interação com metais pesados e substâncias tóxicas, entre outras (SCHNITZER, 1991). Além disso, a adição de MO ao solo construído pode minimizar as emissões do metano resultante da decomposição dos RSU (ABICHOU et al., 2006), bem como melhorar o desenvolvimento da vegetação a ser implantada (RAWLINSON et al., 2004).

As espécies mais recomendadas para a vegetação do solo construído sobre o aterro encerrado são aquelas nativas da região, as quais passaram por um processo de seleção ao longo de milhares de anos e são mais adaptadas a distúrbios ecológicos (WAUGH, 1994). Entretanto, é necessário manter uma diversidade mínima de espécies, possibilitando melhor ciclagem de nutrientes e maior remoção de água via evapotranspiração (LINK; WAUGH; DOWNS, 1994). A implantação de espécies arbóreas pode ainda não ser recomendada (SIMMONS, 1999), pois o seu desenvolvimento pode ser comprometido sobremaneira devido à compactação e pequena espessura do

Tabela 2 - Modelos ${ }^{1}$ e parâmetros² estimados do semivariograma para os atributos do solo construído

\begin{tabular}{|c|c|c|c|c|c|c|c|c|}
\hline \multirow{2}{*}{ Parâmetro } & Areia & Silte & Argila & \multirow{2}{*}{$\mathrm{pH}$} & MO & V\% & $\mathrm{Al} \%$ & $P$ \\
\hline & & $\mathrm{g} \mathrm{kg}^{-1}$ & & & \multicolumn{3}{|c|}{$\%$} & $\mathrm{mg} \mathrm{kg}^{-1}$ \\
\hline Modelo & Gauss & Esf & Exp & Gauss & Gauss & Gauss & Esf & Gauss \\
\hline $\mathrm{C}_{0}$ & 480 & 6,5 & 290 & $1,0 \mathrm{E}-4$ & $2,1 \mathrm{E}-3$ & 0,1 & 3,5 & 0,01 \\
\hline $\mathrm{C}_{0}+\mathrm{C}$ & 5084 & 293,3 & 5860 & 0,22 & 0,06 & 135,5 & 188,2 & 4,27 \\
\hline$A(m)$ & 52,1 & 43,0 & 133,2 & 36,4 & 34,3 & 32,2 & 36,5 & 36,7 \\
\hline IDE (\%) & 9 & 2 & 5 & 0 & 4 & 0 & 2 & 0 \\
\hline$r^{2}$ & 0,94 & 0,7 & 0,95 & 0,24 & 0,63 & 0,29 & 0,55 & 0,42 \\
\hline SQR & $5,1 \mathrm{E}+5$ & $2,8 \mathrm{E}+3$ & $3,8 \mathrm{E}+5$ & $3,3 \mathrm{E}-2$ & $1,3 \mathrm{E}-4$ & $4,0 \mathrm{E}+3$ & $1,1 \mathrm{E}+3$ & 4,42 \\
\hline EP & $-0,12$ & 0,35 & 0,41 & 0,74 & $-0,19$ & 0,51 & $-0,56$ & 0,96 \\
\hline CV (\%) & 48 & 41 & 89 & 141 & 44 & 25 & 27 & 20 \\
\hline W & $0,70 \mathrm{~ns}$ & $0,95 a b$ & 0,69 ns & 0,60 ns & $0,94 \mathrm{a}$ & $0,93 \mathrm{~ns}$ & $0,96 a b$ & $0,97 a b$ \\
\hline \multirow{2}{*}{ Parâmetro } & $\mathrm{Ca}^{2+}$ & $\mathrm{Mg}^{2+}$ & $\mathrm{K}^{+}$ & $\mathrm{Na}^{+}$ & $\mathrm{Al}^{3+}$ & $\mathrm{H}^{+}+\mathrm{Al}^{3+}$ & $\mathrm{CTC}_{\text {efetiva }}$ & CTC $_{\text {potencial }}$ \\
\hline & \multicolumn{8}{|c|}{$\mathrm{cmol}_{\mathrm{c}} \mathrm{kg}^{-1}$} \\
\hline Modelo & Linear & Gauss & Gauss & Gauss & Exp & Gauss & Gauss & Gauss \\
\hline $\mathrm{C}_{0}$ & 0,19 & $1,5 \mathrm{E}-3$ & $1,0 \mathrm{E}-5$ & $1,0 \mathrm{E}-5$ & 0,11 & 0,11 & $1,0 \mathrm{E}-3$ & $1,0 \mathrm{E}-3$ \\
\hline $\mathrm{C}_{0}+\mathrm{C}$ & 0,19 & 0,29 & $1,9 \mathrm{E}-2$ & $3,0 \mathrm{E}-2$ & 0,58 & 0,84 & 1,3 & 1,53 \\
\hline$A(m)$ & 118,0 & 34,0 & 44,9 & 53,0 & 50,4 & 36,4 & 37,9 & 42,4 \\
\hline IDE (\%) & 100 & 1 & 0 & 0 & 18 & 13 & 0 & 0 \\
\hline$r^{2}$ & 0,44 & 0,78 & 0,57 & 0,82 & 0,53 & 0,87 & 0,82 & 0,91 \\
\hline SQR & $4,1 \mathrm{E}-3$ & $1,7 \mathrm{E}-3$ & $9,4 \mathrm{E}-5$ & $9,1 \mathrm{E}-5$ & $1,0 \mathrm{E}-2$ & $8,7 \mathrm{E}-3$ & $5,1 \mathrm{E}-2$ & $4,8 \mathrm{E}-2$ \\
\hline EP & 0,79 & 0,55 & 0,79 & 0,91 & $-0,31$ & $-0,55$ & 0,48 & 0,46 \\
\hline CV (\%) & 12 & 10 & 29 & 9 & 24 & 28 & 35 & 110 \\
\hline$W^{2}$ & $0,97 a b$ & $0,97 a b$ & $0,96 a b$ & $0,73 \mathrm{~ns}$ & $0,96 a b$ & $0,82 \mathrm{~ns}$ & $0,93 \mathrm{~ns}$ & $0,62 \mathrm{~ns}$ \\
\hline
\end{tabular}

1Modelos: Gauss - gaussiano; Exp - exponencial; Esf - esférico; Linear - linear.

2 Parâmetros: $\mathrm{C}_{0}$ - efeito pepita; $\mathrm{C}_{0}+\mathrm{C}$ - patamar; A - alcance; IDE - índice de dependência espacial; $\mathrm{r}^{2}$ - coeficiente de determinação; SQR - soma do quadrado dos resíduos; EP coeficiente de correlação de Pearson (r) para o efeito proporcional entre média e variabilidade locais; CV - coeficiente de variação; W - estatística do teste de normalidade de Shapiro-Wilk. Valores seguidos pelas letras "a" e "b" indicam normalidade dos dados com nível de significância de 1 e 5\%, respectivamente; ns - não-significativo em quaisquer níveis de significância. 
solo, presença de chorume em subsuperfície, alto $\mathrm{pH}$ em alguns pontos e baixas concentrações de oxigênio (DOBSON; MOFFAT, 1995; DICKINSON, 2000; RAWLINSON et al., 2004).

\section{Conclusões}

O solo construído apresenta inúmeras restrições ao desenvolvimento da vegetação. A utilização da geoestatística para mapear os atributos do solo foi ineficiente, considerando a malha amostral utilizada. A redução do tamanho da malha amostral necessária para tornar possível o uso da geoestatística elevaria os custos do trabalho. Portanto, a melhor alternativa é a utilização de ferramentas de análise multivariada de dados para definição de zonas homogêneas de manejo. Em conjunto, os atributos teor de areia (ou argila), saturação por bases e pH foram capazes de explicar a estrutura da variabilidade do solo construído. Isso é importante devido à facilidade de determinação destes atributos em laboratório, da sua relação com o processo de cobertura dos RSU, além de serem indicativos da saturação do solo com chorume e, ainda, determinarem aspectos relacionados ao desenvolvimento da vegetação. Portanto, tais atributos podem ser dados como prioritários no estabelecimento de ações de intervenção com vistas à remediação de solos construídos sobre aterros encerrados de RSU.

\section{Agradecimentos}

Ao Conselho Nacional de Desenvolvimento Científico e Tecnológico, pelas bolsas concedidas, e aos revisores e ao corpo editorial da RESA, pelas sugestões para a melhoria do trabalho.
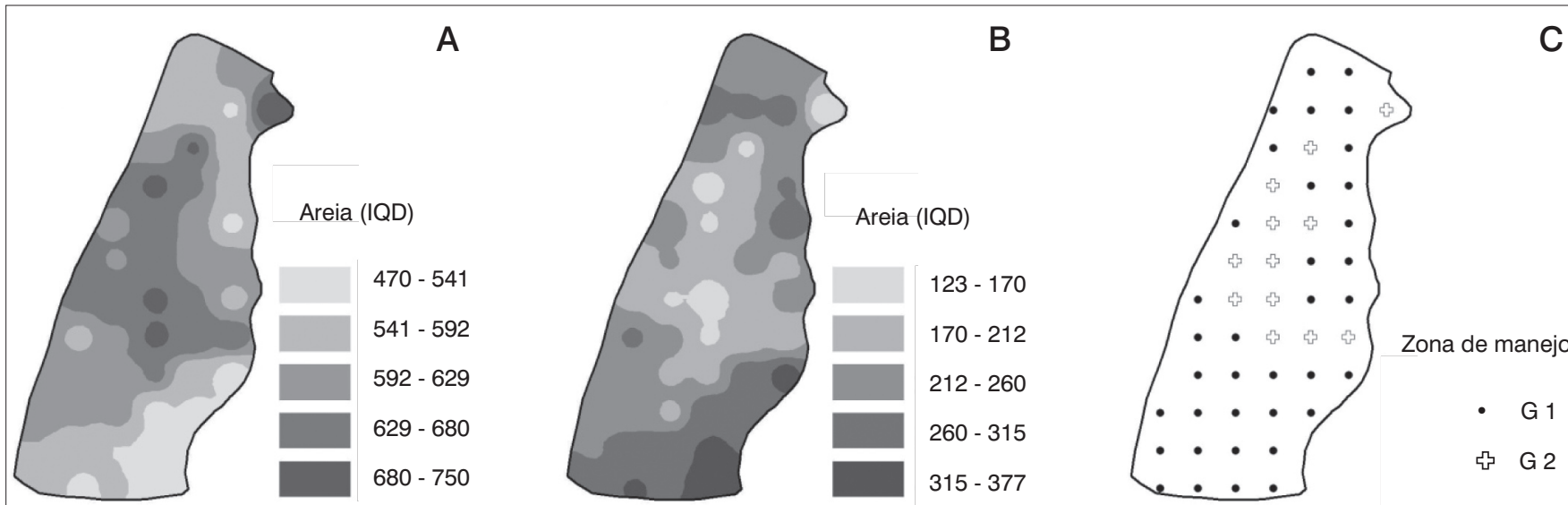

Figura 1 - Mapas do teor de areia (a) e argila (b) $\left(\mathrm{g} \mathrm{kg}^{-1}\right)$ e zonas de manejo (c) do solo construído.

Tabela 3 - Estatísticas descritivas dos atributos ${ }^{1,2}$ do solo construído para as zonas de manejo $G_{1}$ e $G_{2}$

\begin{tabular}{|c|c|c|c|c|c|c|c|c|}
\hline \multirow{2}{*}{ Estatística } & Areia & Silte & Argila & \multirow{2}{*}{$\mathrm{pH}$} & MO & V\% & $\mathrm{Al} \%$ & $\mathbf{P}$ \\
\hline & & $\mathrm{g} \mathrm{kg}^{-1}$ & & & \multicolumn{3}{|c|}{$\%$} & $\mathrm{mg} \mathrm{kg}^{-1}$ \\
\hline \multicolumn{9}{|c|}{ Zona de manejo $\mathrm{G}_{1}$} \\
\hline Média & 570 & 164 & 266 & 4,8 & 1,0 & 39 & 44 & 1,9 \\
\hline Máximo & 645 & 205 & 378 & 5,3 & 1,5 & 73 & 60 & 11,4 \\
\hline \multicolumn{9}{|c|}{ Zona de manejo $G_{2}$} \\
\hline Média & 685 & 156 & 158 & 5,3 & 1,1 & 51 & 22 & 2,6 \\
\hline Máximo & 751 & 197 & 221 & 7,3 & 1,3 & 94 & 36 & 7,7 \\
\hline \multirow{2}{*}{ Estatística } & $\mathrm{Ca}^{2+}$ & $\mathrm{Mg}^{2+}$ & $\mathrm{K}^{+}$ & $\mathrm{Na}^{+}$ & $\mathrm{Al}^{3+}$ & $\mathrm{H}^{+}+\mathrm{Al}^{3+}$ & CTC $_{\text {efetiva }}$ & CTC $\mathrm{Potencial}$ \\
\hline & \multicolumn{8}{|c|}{$\mathrm{cmol}^{\mathrm{kg}^{-1}}$} \\
\hline \multicolumn{9}{|c|}{ Zona de manejo $\mathrm{G}_{1}$} \\
\hline Média & 1,0 & 1,4 & 0,12 & 0,07 & 2,0 & 4,0 & 4,6 & 6,6 \\
\hline Mínimo & 0,5 & 0,7 & 0,07 & 0,03 & 0,1 & 0,4 & 2,4 & 4,0 \\
\hline Máximo & 2,1 & 2,3 & 0,80 & 0,73 & 1,7 & 3,6 & 5,6 & 7,3 \\
\hline
\end{tabular}




\section{Referências}

$\mathrm{ABICHOU}, \mathrm{T}$. et al. Methane flux and oxidation at two types of intermediate landfill covers. Waste Management, v.26, p.1305-1312, 2006

CAMPOS, M.L.; ALMEIDA, J.A.; SOUZA, L.S. Avaliação de três áreas de solo construído após mineração de carvão a céu aberto em Lauro Müller, Santa Catarina. Revista Brasileira de Ciência do Solo, v.27, p.1123-1137, 2003.

CHAVES, L.H.G. et al. Avaliação da salinidade dos Neossolos dos perímetros irrigados de São Gonçalo e Engenheiro Arcoverde, PB. Agropecuária Técnica, v.26, p.15-21, 2005.

CRITTO, A.; CARLON, C.; MARCOMINI, A. Characterization of contaminated soil and groundwater surrounding an illegal landfill (S. Giuliano, Venice, Italy) by principal component analysis and kriging. Environmental Pollution, v.122, p.235-244, 2003.

DEDYSH, S.N.; PANIKOV, N.; TIEDJE, J.M. Acidophilic methanotrophic communities from sphagnum peat bogs. Applied and Environmental Microbiology, v.64, p. 922-929, 1998

DICKINSON, N. Strategies for sustainable woodland on contaminated soils. Chemosphere, v.41, p.259-263, 2000.

DOBSON, M.C.; MOFFAT, A.J. Site capability assessment for woodland creation on landfills. Farnham: Forestry Authority Research Information (Note 263), 1995.

DUDAL, R.; NACHTERGAELE, F.; PURNELL, M. The human factor of soil formation. In: WORLD CONGRESS OF SOIL SCIENCE, 17, 2002, Bangkok, Thailand. Anais... Bangkok: International Union of Soil Science, 2002.

DWYER, S.F. Landfill covers: is it time to change current regulations? Albuquerque: Sandia National Laboratories, 2000. 25p.

EMPRESA BRASILEIRA DE PESQUISA AGROPECUÁRIA - EMBRAPA. Manual de métodos de análise de solos. 2. ed. Rio de Janeiro: EMBRAPA, 1997. $221 \mathrm{p}$

FRANTZIS, I. Settlement in the landfill site of Schisto. In: INTERNATIONAL LANDFILL SYMPOSIUM, 3., 1991, S. Margarita Di Pula, Sardenha, Itália. Anais... Cagliari: CISA, 1991. p. 1189-1195.

GUILLEN, D.P.; HERTZOG, R.C. A Survey of Department of Energy sponsored geophysical research for shallow waste site characterization. Vadose Zone Journal, v.3, p.122-133, 2004.

ISAAKS, E.H.; SRIVASTAVA, M. An introduction to applied geostatistics. New York: Oxford University Press, 1989. 600p.

KÄMPF, N.; SCHNEIDER, P.; GIASSON, E. Propriedades, pedogênese e classificação de solos construídos em áreas de mineração na bacia carbonífera do baixo Jacuí (RS). Revista Brasileira de Ciência do Solo, v.21, p.79-88, 1997.

KERRY, R.; OLIVER, M.A. Average variograms to guide soil sampling. International Journal of Applied Earth Observation and Geoinformation, v.5, p. 307-325, 2004

LAUERMANN, A. Caracterização química dos efluentes gerados pelo Aterro Controlado de Santa Maria e retenção de chumbo e zinco por um Argissolo da depressão central do Rio Grande do Sul. 2008. $72 f$ Dissertação (Mestrado em Ciência do Solo) - Universidade Federal de Santa Maria, Santa Maria, RS, 2008.

LINK, S.O.; WAUGH, W.J.; DOWNS, J.L. The role of plants in isolation barrier systems. In: GEE, G.W.; WING, N.R. (Eds.) In-situ remediation: scientific basis for current and future technologies. Richland: Batelle Press, 1994. p. 561-592.

MAÇANEIRO, K.C. Efeito da calagem e da adubação orgânica no estabelecimento de gramíneas em áreas de solo construído após mineração de carvão a céu aberto em Lauro Müller, Santa Catarina. 2001. 66f. Dissertação (Mestrado em Ciência do Solo) - Universidade do Estado de Santa Catarina, Florianópolis, 2001.

McBRATNEY, A.B. et al. A purposive sampling scheme for precision agriculture. In: STAFFORD, J.J. (Ed.). Precision Agriculture '99. Sheffield: Sheffield Academic Press, 1999. p.101-110.

MORENO, J.A. Clima do Rio Grande do Sul. Porto Alegre: Secretaria da Agricultura, Diretoria de Terras e Colonizações, Secção de Geografia, 1961. $46 \mathrm{p}$.

RAWLINSON, $\mathrm{H}$. et al. Woodland establishment on closed old-style landfill sites in N.W., England. Forest Ecology and Management, v.202, p.265-280, 2004.

SAMUEL-ROSA, A.; DALMOLIN, R.S.D. Fauna edáfica em solo construído, campo nativo e lavoura anual. Ciência Rural, v.39, p.913-917, 2009.

SANTOS, R.V.; MURAOKA, T. Interações salinidade e fertilidade do solo. In: GHEYI, H.R.; QUEIROZ, J.E.; MEDEIROS, J.F. (Eds.) Manejo e controle da salinidade na agricultura irrigada. Campina Grande: Universidade Federal da Paraíba, 1997. p. 289-317.

SCHAFER, W.M.; NIELSEN, G.A.; NETTLETON, W.D. Minesoil genesis and morphology in a spoil chronosequence in Montana. Soil Science Society of America Journal, v.44, p.802-807, 1980.

SCHNITZER, M. Soil organic matter - the next 75 years. Soil Science, v.151, p.41-58, 1991

SIMMONS, E. Restoration of landfill sites for ecological diversity. Waste Management \& Research, v.17, p.511-519, 1999.

STEIN, A.; ETTEMA, C. An overview of spatial sampling procedures and experimental design of spatial studies for ecosystem comparisons. Agriculture, Ecosystems \& Environment, v.94, p.31-47, 2003.

TEIXEIRA, C.E. et al. Estudos sobre a oxidação aeróbia do metano na cobertura de três aterros sanitários no Brasil. Engenharia Sanitária \& Ambiental, v.14, n.99-108, 2009.

WAUGH, W.J. Paleoclimatic data application: long-term performance of uranium mill tailings repositories. In: Climate change in the four corners and adjacent regions: implications for environmental restoration and landuse planning, Anais... Colorado: Grand Junction, 1994.

WEBSTER, R.; OLIVER, M.A. Sample adequately to estimate variograms of soil properties. European Journal of Soil Science, v.43, p.177-192, 1992. 